\title{
On the evolution of the Snow Line in Protoplanetary Discs
}

\author{
Rebecca G. Martin ${ }^{1}$ and Mario Livio ${ }^{2}$ \\ ${ }^{1}$ NASA Sagan Fellow, JILA, University of Colorado, Boulder, CO 80309, USA \\ email: rebecca.martin@jila.colorado.edu \\ ${ }^{2}$ Space Telescope Science Institute, 3700 San Martin Drive, Baltimore, MD 21218, USA
}

\begin{abstract}
We examine the evolution of the snow line in a protoplanetary disc. If the magnetorotational instability (MRI) drives turbulence throughout the disc, there is a unique snow line outside of which the disc is icy. The snow line moves closer to the star as the infall accretion rate drops. Because the snow line moves inside the radius of the Earth's orbit, the formation of our water-devoid planet is difficult with this model. However, protoplanetary discs are not likely to be sufficiently ionised to be fully turbulent. A dead zone at the mid-plane slows the flow of material through the disc and a global steady state cannot be achieved. We model the evolution of the snow line also in a disc with a dead zone. As the mass is accumulating, the outer parts of the dead zone become self gravitating, heat the massive disc and thus the outer snow line does not come inside the radius of the Earth's orbit. With this model there is sufficient time and mass in the disc for the Earth to form from water-devoid planetesimals at a radius of $1 \mathrm{AU}$. Furthermore, the additional inner icy region within the dead zone predicted by this model may allow for the formation of giant planets close to their host star without the need for much migration.
\end{abstract}

Keywords. Earth - planetary systems: protoplanetary discs - planets and satellites: formation

\section{Introduction}

The inner terrestrial planets have very little water compared to the outer giant planets. The current water content of the Earth is $0.023 \%$ by weight compared to the outer planets that have greater than $40 \%$. In the solar nebula, ice forms beyond the snow line radius, $R_{\text {snow }}$. The snow line in our solar system is currently observed to be within the asteroid belt. Thus, at the time of planetesimal formation, the snow line was at a radius of $R=2.7$ AU from the Sun. Outside of the snow line, planetesimal formation is much easier because of the increased solid density. Thus, we expect massive planets to form outside of the snow line. Fully turbulent disc models predict a minimum snow line radius that is inside the orbital radius of the Earth. With this model it is hard to explain the formation of our water devoid Earth. However, protoplanetary discs are not likely to be fully ionised. They contain a dead zone about the mid-plane where the MRI cannot drive turbulence. We examine this disc model here.

\section{Protoplanetary Discs with Dead Zones}

Dead zones form when the ionisation fraction is too low for the magnetic field to be well coupled to the disc (Gammie 1996). The inner parts of the disc have a temperature higher than the critical required for complete thermal ionisation, $T_{\mathrm{c}}>T_{\text {crit }}$. Further out, the magneto-rotational instability (MRI) is suppressed except in the surface layers of the disc that are ionised by cosmic rays or X-rays. In the low surface density outer parts of the disc, where $\Sigma<\Sigma_{\text {crit }}$, cosmic rays can penetrate and ionise the whole disc. 


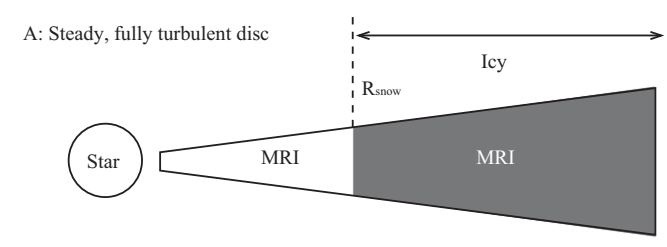

B: Time-dependent disc with a dead zone
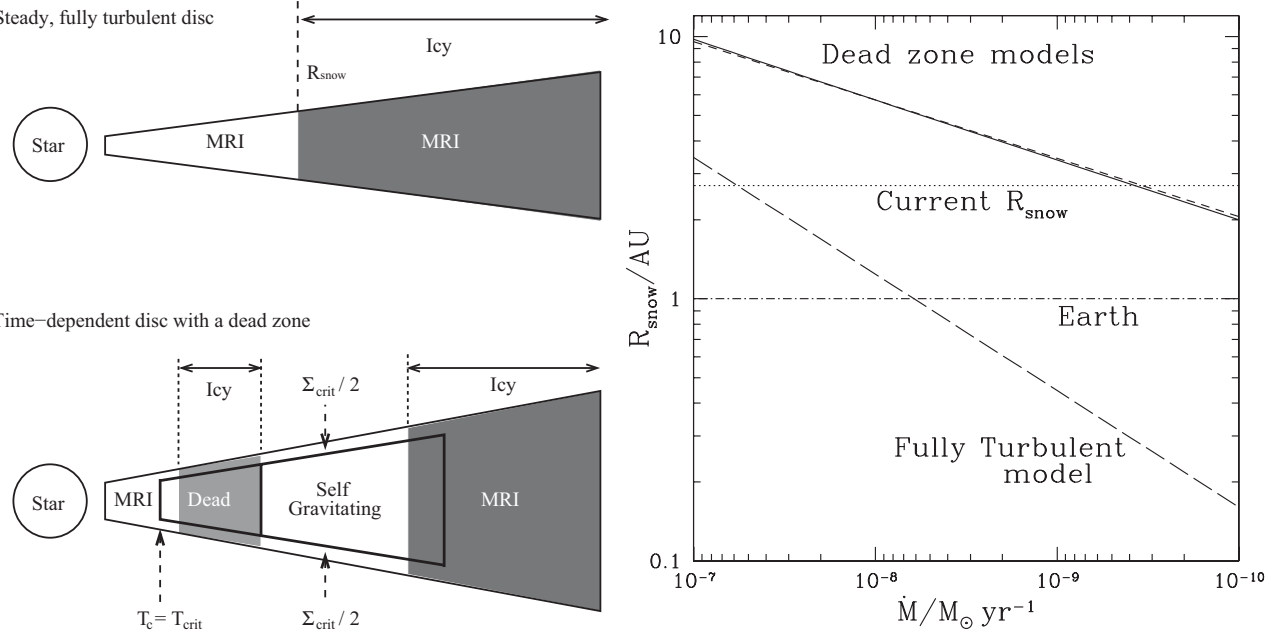

Figure 1. Left: Sketches of the protoplanetary disc structure with and without a dead zone. Right: The snow line radius as a function of infall accretion rate.

Material builds up in the dead zone until the outer parts become self gravitating (see Fig. 1) and this drives some extra turbulence and heating within the disc. In the early stages of disc evolution, this can lead to transitions between gravitationally and magnetically produced turbulence and outbursts on to the young star (e.g. Armitage et al. 2001, Martin \& Lubow 2011). In the later stages when the infall accretion rate has dropped, the outbursts cease but a dead zone may still be present. The dead zone changes the temperature profile of a disc significantly from the fully turbulent model.

We find an analytic solution for the outermost snow line radius in a disc with a dead zone (Martin \& Livio 2013). We find good agreement with the one-dimensional timedependent layered accretion disc models (Martin \& Livio 2012, Martin et al. 2012). In the disc with a dead zone, the extra gravitational turbulence heats the disc and the outer icy region remains outside the orbital radius of the Earth. Thus, the formation of a water devoid Earth at $R=1 \mathrm{AU}$ is possible.

\section{Conclusions}

In a fully turbulent disc, the unique snow line passes inside the radius of the Earth's orbit causing problems for the formation of our water-devoid planet Earth. When a dead zone forms in the disc, it prevents mass flow through the disc and the outer regions become self-gravitating. The turbulence driven by self-gravity increases the temperature of the outer parts of the dead zone and thus the outer icy region is much farther from the star. On a more speculative note, the inner icy region within the dead zone, not predicted by the turbulent disc model, could allow for the formation of icy planets or gas giants in the inner regions of exo-solar systems if there is water present there.

\section{References}

Armitage P. J., Livio M., \& Pringle J. E., 2001, MNRAS, 324, 705

Gammie C. F., 1996, ApJ, 457, 355

Martin R. G. \& Lubow S. H., 2011, ApJ, 740, 6

Martin R. G., Lubow S. H., Livio M., \& Pringle J. E., 2012, MNRAS, 423, 2718

Martin R. G. \& Livio M., 2012, MNRAS, 425, L6

Martin R. G. \& Livio M., 2013, MNRAS, 434, 633 